\title{
Derivation of Green's Function Representations for the Analysis of Sectoral Waveguides Embedded in Cylindrically Stratified Media
}

\author{
M. Kalfa, ${ }^{1,2}$ and V.B. Erturk ${ }^{1 *}$ \\ ${ }^{1}$ Dept. of Electrical and Electronics Engineering, \\ Bilkent University, TR-06800, Bilkent, Ankara, Turkey \\ ${ }^{2}$ Radar, Electronic Warfare, and Intelligence Systems Division, \\ Aselsan Inc., TR-06370, Ankara, Turkey
}

The design and analysis of dielectric-covered slotted waveguide arrays are of great interest in many military and civil applications, because of their low-profile, high efficiency and high power handling capabilities. Regarding the efficient and accurate analysis of such antennas, integral equation (IE) based methods that utilize the method of moments (MoM) or a hybrid combination of MoM with an appropriate Green's function (referred to as MoM/Green's function technique) are widely used. For the hybrid MoM/Green's function technique, appropriate dyadic Green's function representations for the waveguide interior and the exterior stratified media are required. However, in the case of a cylindrically conformal dielectric-covered slotted waveguide array, which may be desired due to aerodynamic and/or radar cross section (RCS) concerns, the abovementioned IE-based analysis that utilize a hybrid MoM/Green's function technique becomes a greater challenge due to the difficulties in the evaluation of the entries of MoM impedance/admittance matrices, especially for the terms related to cylindrically stratified media.

Because the accuracy and efficiency of the abovementioned analysis method strongly depend on the efficiency and accuracy of the used Green's function representations, in this study, the Green's function representations for the two solution regions are rigorously derived and available acceleration algorithms are implemented during their numerical evaluations. For the waveguide interior, the Green's function representations for longitudinal slots on the sectoral waveguide are required. Hence, first the longitudinal slots are represented with magnetic current modes and then the appropriate Green's function representations are derived for the longitudinal component of the electric vector potential $(\vec{F})$. Then, the corresponding magnetic field component of the aforementioned Green's function representation is found.

Regarding the exterior cylindrically stratified medium (that may represent monolithic or sandwich radomes), spectral domain Green's function representations are readily available in literature. These functions are modified in such a way that all Bessel (Hankel) functions are in the form of ratios of two Bessel (Hankel) functions, which allows utilization of asymptotic approximations that leads to the efficient summation of eigenmodes. The computed spectral domain expressions are represented in the form of discrete complex images (DCIM) via the generalized pencil of function (GPOF) method and the final space domain transform integrals are taken analytically that yield the form of closed-form Green's function representations (CFGF). The singular terms related to the paraxial and the source regions are treated analytically during the mutual admittance calculations.

The derived Green's functions are computed for various source and observation points, and the results are compared to the results obtained from the commercially available full-wave electromagnetic solvers. 